\title{
Agarose-stabilized gold nanoparticles for surface-enhanced Raman spectroscopic detection of DNA nucleosides
}

\author{
V. Kattumuri, M. Chandrasekhar, and S. Guha ${ }^{a}$ \\ Department of Physics and Astronomy, University of Missouri, Columbia, Missouri 65211 \\ K. Raghuraman and K. V. Katti \\ Department of Radiology, University of Missouri, Columbia, Missouri 65211 \\ K. Ghosh and R. J. Patel \\ Department of Physics and CASE, Missouri State University, Springfield, Missouri 65804
}

(Received 5 July 2005; accepted 9 March 2006; published online 12 April 2006)

\begin{abstract}
We present surface-enhanced Raman scattering (SERS) studies of DNA nucleosides using biologically benign agarose-stabilized gold nanoparticles (AAuNP). We compare the SERS activity of nucleosides with AAuNP to that of commercially obtained citrate-stabilized gold nanoparticles and find the SERS activity to be an order of magnitude higher with AAuNP. The higher SERS activity is explained in terms of the agarose matrix, which provides pathways for the gold nanoparticles to have distinct arrangements that result in stronger internal plasmon resonances.

(C) 2006 American Institute of Physics. [DOI: 10.1063/1.2192573]
\end{abstract}

Nanoparticle research is at the leading edge of a rapidly growing technology in nanoscale, medical, and biological sciences. Advances in the preparation of gold and silver nanoparticles have rekindled interest in surface-enhanced Raman scattering (SERS) as a means to detect single molecules and to identify their chemical structure; SERS now provides a potential for label-free detection of biomolecules. ${ }^{1}$ Significant efforts focus on the binding of oligonucleosides to metal surfaces and colloids for applications that include multiplexed DNA detection technology, ${ }^{2}$ rapid sequencers based on SERS from single DNA bases, ${ }^{3}$ and real-time DNA detection methodology. ${ }^{4}$

In this work we use biologically benign agarosestabilized gold nanoparticles for the detection of micromolar concentrations of DNA nucleosides using SERS. This process for gold nanoparticle production allows for systematic variation in nanoparticulate size via changes in the stoichiometry of the precursors. Agarose-stabilized gold nano $<-$ ? $>$ particles have several advantages over the conventional citrate reduction technique of preparing nanoparticles; agarose, which is a biologically benign medium, ensures nondegradation of probe molecules. Furthermore, its gelation properties facilitate easy film formation for on-chip biosensing applications.

Although the relative importance of various mechanisms for SERS is still debated, electromagnetic enhancement due to the local field of surface plasmons excited by the incident light is considered to have a major contribution. ${ }^{3}$ A major enhancement occurs for aggregated colloidal silver or gold nanoparticles (induced by halide ions), where plasmon resonances can result in a strong confinement of the optical fields between the aggregated particles. ${ }^{3}$ Another mechanism involves dynamical charge transfer between a nanoparticle and a molecule, or a resonance Raman effect due to a chargetransfer electronic transition. The magnitudes of such chemical enhancement have been estimated to reach factors only of the order of $10-100 .^{5}$ It was pointed out by Haslett et al. that

\footnotetext{
a)Electronic mail: guhas@missouri.edu
}

a chemical contribution to SERS almost always exists for chemisorbed molecules but that alone cannot explain the existence of SERS. ${ }^{6}$

Estimates of enhancement factors based on the comparison of SERS intensity with normal Raman intensity are usually tricky since the number of target molecules that contribute to the SERS signal is usually unknown. The largest SERS enhancements reported in the literature occur when excitation wavelengths are in the near infrared (NIR), out of resonance with the surface plasmons. This effect has been reported in a series of papers by Kneipp et al.; ${ }^{7,8}$ they estimate the SERS cross section by comparing the intensities of the surface-enhanced anti-Stokes $\left(I_{\text {as }}\right)$ to the Stokes Raman scattering $\left(I_{s}\right)$ signal. Recently it was shown that the $I_{\text {as }} / I_{s}$ ratio for a specific mode in a sample is dependent upon the laser wavelength, a manifestation of the internal or the local plasmon resonance due to the presence of "hot spots," where two nanoparticles are most strongly coupled. ${ }^{9}$ Hot spots result in the redshift of local plasmon resonances and SERS signals that are higher when the excitation wavelength is off the surface plasmon resonance. ${ }^{10} \mathrm{~A}$ scale invariant theory of Raman scattering of light by fractal clusters further shows that more hot spots are excited when the laser wavelength is in the NIR. ${ }^{11}$ In our measurements we do not use the $I_{\text {as }} / I_{s}$ ratio, but instead compare the SERS Stokes Raman signal to that of the substrate Si Raman phonon. This provides an excellent way of taking into account thickness variations between the different sample films (described later).

The agarose-stabilized gold nanoparticles (AAuNP) were produced by reduction of $\mathrm{NaAuCl}_{4}$ by trimeric alanine phosphine conjugate (THPAL, $\left.\mathrm{P}\left(\mathrm{CH}_{2} \mathrm{NHCH}_{3} \mathrm{COOH}\right)_{3}\right)$ in the presence of agarose in water at $25^{\circ} \mathrm{C}$ and at physiological $p \mathrm{H}$ values. The size distribution can be varied by altering the concentration of either gold ions or reducing agent. Specifically, at any given concentration of gold in agarose gel matrix, increasing the concentration of the reducing agent yielded larger nanoparticles. This is, presumably, because of the sudden increase in the local concentration of the reducing agent around gold ions inside the gel matrix (published 

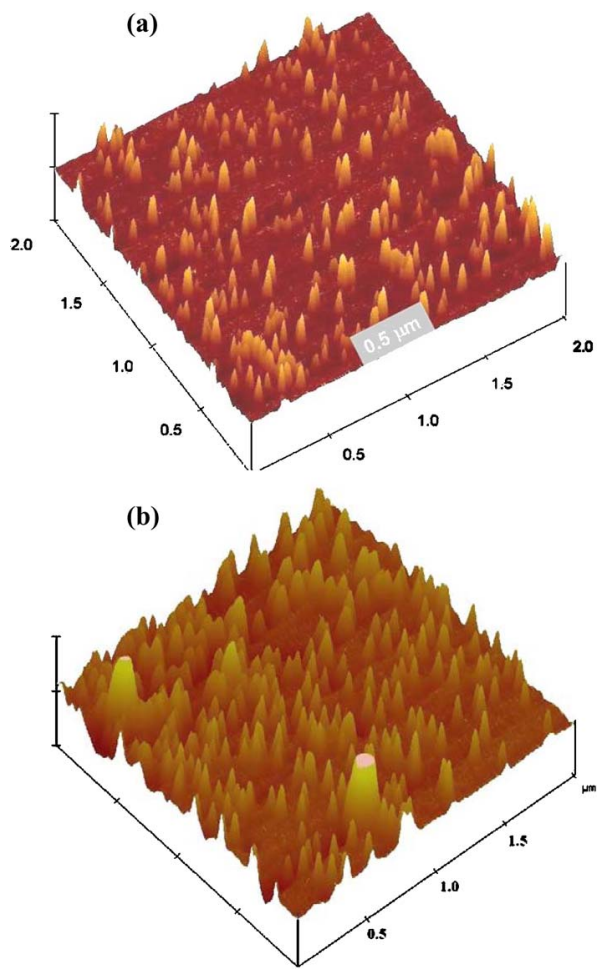

FIG. 1. (Color online) (a) AFM image of a drop-casted AAuNP film on a quartz substrate. (b) AFM image of a drop-casted CAuNP film on a quartz substrate.

elsewhere). ${ }^{12}$ Citrate-stabilized gold nanoparticles (CAuNP), with mean particulate size of $15 \mathrm{~nm}$, were commercially obtained from Aldrich. DNA nucleosides 2'-deoxyadenosine (dA), 2'-deoxycytidine-5' -monophosphoric acid (dCMP), and 2'-deoxyguanosine-5'-monophosphate (dGMP) were procured from Acros, and 2'-deoxythymidine (dT) was obtained from Aldrich. To each of the DNA nucleosides, we added $0.1 \mathrm{ml}$ of $5 \times 10^{-3} M$ base solution to $1 \mathrm{ml}$ of AuNP for a final nucleoside concentration of $\sim 10^{-4} M$. The films were prepared by drop casting $30-50 \mu \mathrm{l}$ of the final solution on Si wafers and drying it in a desiccator for 12-20 h.

Raman spectra were collected by an Invia Renishaw spectrometer. The $785 \mathrm{~nm}$ line of a diode laser $(\sim 5 \mathrm{~mW})$ was used as the excitation source. Atomic force microscopy (AFM) measurements were performed in the tapping mode with a Nanoscope III system and absorption measurements were carried out using a Shimadzu UV-vis spectrophotometer.

Figure 1(a) shows a $2 \times 2 \mu \mathrm{m}^{2}$ AFM image of a thin film cast from AAuNP on a quartz substrate. Since agarose forms a gel matrix, it is necessary to slightly heat the films to get a good contrast. The AuNPs are uniformly distributed over the substrate with a submonolayer coverage and a sharp size distribution. For comparison, an AFM image of the CAuNP film is shown in Fig. 1(b). The particle size in the AFM images, $\sim 35 \mathrm{~nm}$, is somewhat larger than that from transmission electron microscope measurements $(\sim 15 \mathrm{~nm})$. This magnification is attributed to the convolution of true particle size with that of the AFM tip. ${ }^{13}$ There are slight variations in the distribution of particles from one film to the other, but overall AAuNP and CAuNP films show a similar number of particles per unit area.

The size distribution of the gold nanoparticles in AAuNP is, however, higher than in CAuNP as seen in their respective absorption spectra (Fig. 2) (intensities are unnormalized). Figure 2(b) shows the UV-vis absorption spectra of AAuNP in the presence of DNA nucleosides. The absorption spectrum of the gold nanoparticles is shown as a reference, where the plasmon band peaks at $\sim 541 \mathrm{~nm}$ and the width of the plasmon band is $\sim 100 \mathrm{~nm}$. The rising background in the lower wavelength region is due to interband transitions in gold. ${ }^{14}$ With the addition of dA, dCMP, and dGMP the plasmon band redshifts by almost $15 \mathrm{~nm}$, and the width of the band also increases by $\sim 30 \mathrm{~nm}$. In dCMP an aggregate peak
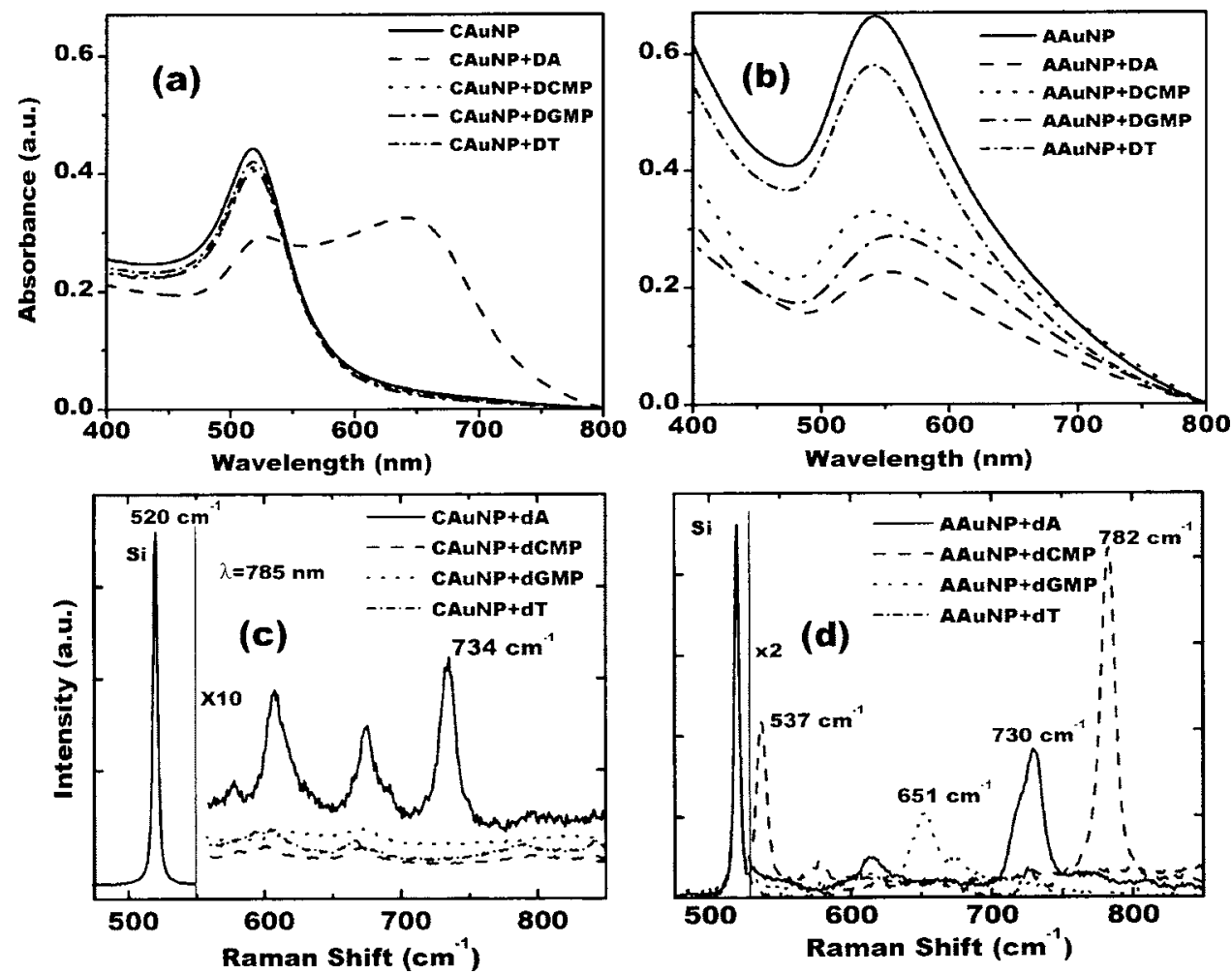

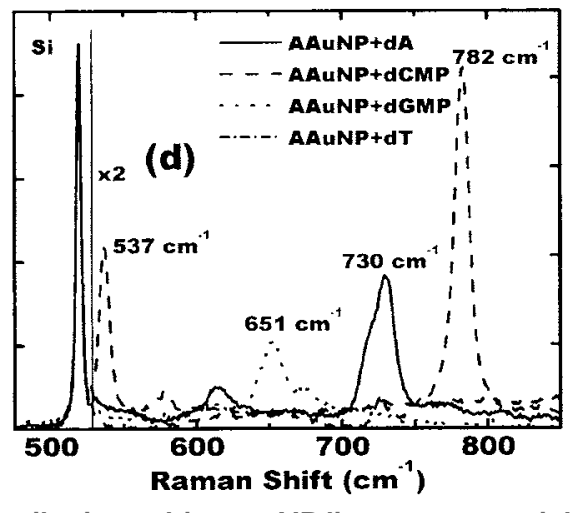

FIG. 2. (a) Absorption spectra of CAuNP and CAuNP with the four DNA nucleosides. (b) Absorption spectra of AAuNP and AAuNP with the four DNA nucleosides. (c) SERS spectra of dA, dCMP, dGMP, and dT with CAuNP. The vertical scale has been expanded by $\times 10$ beyond $550 \mathrm{~cm}^{-1}$. (d) SERS spectra of $\mathrm{dA}$, dCMP, dGMP, and dT with AAuNP. The vertical scale has been expanded by $\times 2$ beyond $526 \mathrm{~cm}^{-1}$. In (c) and (d) the spectra have been scaled so that the $520 \mathrm{~cm}^{-1} \mathrm{Si}$ peak observed through the sample has the same intensity for all four samples. 
TABLE I. SERS intensity of the DNA nucleosides. The intensities have been normalized to the thickness of the sample as described in the text.

\begin{tabular}{lcc}
\hline \hline & $\operatorname{AAuNP}\left(I_{\text {SERS }}\right)$ & $\operatorname{CAuNP}\left(I_{\text {SERS }}\right)$ \\
\hline $\mathrm{dA}$ & $I_{730}=2.5 \times 10^{5}$ & $I_{734}=0.5 \times 10^{5}$ \\
$\mathrm{dCMP}$ & $I_{782}=10 \times 10^{5}$ & No SERS \\
$\mathrm{dGMP}$ & $I_{651}=1.1 \times 10^{5}$ & No SERS \\
$\mathrm{dT}$ & No SERS & No SERS \\
\hline \hline
\end{tabular}

appears as a shoulder at $650 \mathrm{~nm}$. dT, on the other hand, shows no major changes in the plasmon band.

Figure 2(a) shows the absorption spectra of CAuNP and CAuNP in the presence of the nucleosides; the plasmon band peaks at $518 \mathrm{~nm}$. dA + CAuNP shifts and exhibits an aggregate peak at $640 \mathrm{~nm}$. Adding dT, dCMP, and dGMP to CAuNP results in almost no change in the plasmon band.

Figure 2(d) displays typical SERS spectra of the four nucleosides with AAuNP at room temperature. In $\mathrm{dA}$ the pyrimidine ring breathing mode at $730 \mathrm{~cm}^{-1}$ is strongly enhanced. In dCMP the same mode shifts to a higher frequency of $782 \mathrm{~cm}^{-1}$, and the $537 \mathrm{~cm}^{-1}$ mode, which corresponds to an asymmetric stretch of the pyrimidine ring, is also enhanced. In dGMP the imidazole ring breathing mode at $650 \mathrm{~cm}^{-1}$ gets enhanced. This band is shifted by $\sim 20 \mathrm{~cm}^{-1}$ to higher wave numbers in saturated aqueous solutions. ${ }^{15} \mathrm{dT}$ shows no SERS, as observed in other work. ${ }^{3}$ The Raman spectra of the four nucleosides with CAuNP were measured from films cast on $\mathrm{Si}$, as shown in Fig. 2(c). Of the four nucleosides, only dA shows SERS activity with enhancement of the pyrimidine ring breathing mode at $734 \mathrm{~cm}^{-1}$.

Normalized intensities of the strongest Raman peak observed in the SERS spectra of the DNA nucleosides are tabulated in Table I. These intensities have been normalized to the thickness of the films in the following manner. By dividing the Raman intensity of the outside Si peak (at $520 \mathrm{~cm}^{-1}$ ) to that of the Si Raman peak obtained through the nucleoside films, we obtain the optical density (OD) of the film (BeerLambert's law). With the assumption that the absorption coefficients are similar for all four bases, on dividing the SERS intensity by the OD, we deduce the normalized SERS intensities. The second and the third column represent the intensity ratios for AAuNP and CAuNP, respectively. SERS is observed for three nucleosides with AAuNP, while it is observed only for dA in CAuNP. The largest SERS signal, which is an order of magnitude higher than $\mathrm{dA}$ in CAuNP, is observed from the pyrimidine ring breathing mode of dCMP in AAuNP at $782 \mathrm{~cm}^{-1}$. Since the concentration of the nucleosides is the same for both agarose- and citratestabilized AuNPs, our results clearly show that the agarose matrix facilitates an enhanced SERS activity.

All our Raman measurements were carried out with the $785 \mathrm{~nm}$ line as the excitation source, which is much lower in energy than the surface plasmon band of AuNP. Previous works have alluded to the idea that aggregation is an important factor for observing SERS. ${ }^{15}$ From our results we note that although the $\mathrm{dA}$ shows a higher aggregation with CAuNP as seen from the absorption spectrum, the SERS signal is weaker than with AAuNP. This indicates that in addition to aggregation, the internal local resonances play a large role in SERS activity. We find that some amount of aggregation is necessary for observing SERS. This aggregation is signaled by an increased surface plasmon linewidth upon addition of the DNA nucleosides, which is most probably related to the presence of hot spots. An optimized distribution of hot spots enhances the dipole-dipole interactions among surface plasmons, creating internal local resonances. ${ }^{10}$ The excitations of hot spots are strongly dependent upon the incident laser wavelength. In Ref. 9, Maher et al. use a discrete dipole approximation; they consider a random distribution of 40 colloidal particles (diameter $\sim 20 \mathrm{~nm}$ ) enclosed in a volume of $200 \times 200 \times 200 \mathrm{~nm}^{3}$ for $10^{5}$ different clusters; the enhancement factors with the $633 \mathrm{~nm}$ excitation is $300 \%$ higher than with $514 \mathrm{~nm}$ excitation. Also, if the density of the colloidal particles increases the internal plasmon interactions become delocalized, thus reducing the SERS activity. In light of these calculations we can qualitatively explain the higher SERS activity with the agarose-stabilized AuNP.

Agarose, which forms a gel, develops a network of narrow channels due to the interpenetrating $\mathrm{H}$ bonds between the sugar moieties with pore sizes $\sim 200 \mathrm{~nm} .^{16}$ These nanochannels provide pathways that gold particles can occupy. The AFM image clearly shows that the number of particles is $\sim 50$ in a $200 \times 200 \mathrm{~nm}^{2}$ area, similar to the model of Maher et al. ${ }^{9}$ This turns out to be an optimal number of particles/area for an enhanced internal plasmon resonance to yield a high SERS activity. In contrast, AuNP in a citratestabilized film favors fewer hot spots since there are no real channels for an optimized distribution of the particles.

In conclusion, we have shown that agarose-stabilized gold nanoparticles yield higher SERS activity for DNA nucleosides which opens up possibilities of using this AuNP for on-chip biosensing applications.

This work was supported through the University of Missouri Research Board grants URB04-023 (S.G.) and URB03080 (M.C. and K.V.K.), NSF under Grant No. DMR0413601and the NCI under Grant No. IR0ICA119412-01. The gold nanoparticles were produced and supplied by the University of Missouri Nanoparticle Production Core Facility.

${ }^{1}$ G. L. Liu and L. P. Lee, Appl. Phys. Lett. 87, 074101 (2005).

${ }^{2}$ Y. W. C. Cao, R. Jin, and C. A. Mirkin, Science 297, 1536 (2002).

${ }^{3}$ K. Kneipp, H. Kneipp, I. Itzkan, R. R. Dasari, and M. S. Feld, J. Phys.: Condens. Matter 14, R597 (2002).

${ }^{4}$ R. C. Bailey, J.-M. Nam, C. A. Mirkin, and J. T. Hupp, J. Am. Chem. Soc. 125, 13541 (2003).

${ }^{5}$ A. Otto, in Light scattering in solids IV: Electronic Scattering, Spin Effects, SERS and Morphic Effects, edited by M. Cardona and G. Güntherodt (Springer, Berlin, 1984).

${ }^{6}$ T. L. Haslett, L. Tay, and M. Moskovits, J. Chem. Phys. 113, 1641 (2000). ${ }^{7}$ K. Kneipp, H. Kneipp, I. Itzkan, R. R. Dasari, and M. S. Feld, Chem. Rev. (Washington, D.C.) 99, 2957 (1999).

${ }^{8}$ K. Kneipp, W. Y. Wang, H. Kneipp, L. T. Perlman, I. Itzkan, R. R. Dasari, and M. S. Feld, Phys. Rev. Lett. 78, 1667 (1997).

${ }^{9}$ R. C. Maher, L. F. Cohen, P. Etchegoin, H. J. N. Hartigan, R. J. C. Brown, and J. T. Milton, J. Chem. Phys. 120, 11746 (2004).

${ }^{10}$ P. Etchegoin, L. F. Chen, H. Hartigan, R. J. C. Brown, M. J. T. Milton, and J. C. Gallop, J. Chem. Phys. 119, 5281 (2003).

${ }^{11}$ M. I. Stockman, V. M. Shalaev, M. Moskovits, R. Botet, and T. F. George, Phys. Rev. B 46, 2821 (1992).

${ }^{12}$ K. Raghuraman, K. K. Katti, and K. Katti (unpublished).

${ }^{13}$ D. Keller, Surf. Sci. 253, 353 (1991).

${ }^{14} \mathrm{U}$. Kreibig and M. Vollmer, Optical Properties of Metal Clusters (Springer, Berlin, 1995).

${ }^{15}$ N. H. Jang, Bull. Korean Chem. Soc. 23, 1790 (2002).

${ }^{16}$ J.-Y. Xiong, J. Narayanan, X.-Y. Liu, T. K. Chong, S. B. Chen, and T.-S Chung, J. Phys. Chem. B 109, 5638 (2005). 